\title{
Clinical Characteristics of Rhabdomyolysis in Children : Single Center Experience
}

\section{Yesul Park, M.D. JiYeon Song, M.D. SuYoung Kim, M.D., Ph.D. Seong Heon Kim, M.D.}

Department of Pediatrics, Pusan National University Children's Hospital, Pusan National University School of Medicine, Yangsan, Korea

\section{Corresponding author: \\ Seong Heon Kim, M.D. \\ Department of Pediatrics, Pusan National University Children's Hospital, 20 Geumo- ro, Mulgeum-eup, Yangsan, Goungnam 50612 , Korea \\ Tel: +82-55-360-3163 \\ Fax: +82-55-360-2181 \\ E-mail:pedksh@gmail.com}

Received: 18 September 2018

Revised: 7 October 2018

Accepted: 11 October 2018
Purpose: Rhabdomyolysis is a metabolic disorder in which the content of damaged muscle cells is released into plasma. Its manifestations include asymptomatic, myalgia, gross hematuria, and complications of acute kidney injury. Because of limited data on rhabdomyolysis in children, we performed this study to determine clinical characteristics of rhabdomyolysis in children.

Methods: We retrospectively reviewed the records of patients with rhabdomyolysis who were treated at the Pusan National University Children's hospital from January 2011 to July 2016. The diagnostic criteria were serum myoglobin level of $\geq 80 \mathrm{ng} / \mathrm{mL}$, exclusive of acute myocardial injury, cardiac arrest, and brain damage. Results: Forty-five patients were enrolled; mean age, $116 \pm 68$ months. Of these, 35 were boys and 10 were girls. Twenty-six patients experienced myalgia and 12 patients showed gross hematuria. Among these, seven patients initially had both myalgia and gross hematuria. The most common causes of rhabdomyolysis were infection, physical exertion, prolonged seizures, metabolic abnormalities, and drug addiction. Acute kidney injury (AKI) was the most common complication, followed by disseminated intravascular coagulation. Thirty-seven patients improved with sufficient fluid supply but two patients underwent hemodialysis due to deterioration of kidney function. Gross hematuria, positive occult blood test, and positive urine protein were more common in patients with AKI than in those without AKI.

Conclusions: In children, infection was the most common cause of rhabdomyolysis. Most patients recovered by sufficient fluid therapy. However, in severe cases, especially in patients with underlying kidney disease, hemodialysis may be neces sary in the present study.

Key words: Rhabdomyolysis, Influenza, Physical exertion, Acute kidney injury, Children

\section{Introduction}

Rhabdomyolysis is a syndrome characterized by rapid breakdown and leakage of skeletal muscle cell contents, such as electrolytes, myoglobin, and other sarcoplasmic proteins [e.g., creatine kinase (CK), aldolase, lactate dehydrogenase (LDH), alanine aminotransferase (ALT), and aspartate aminotransferase (AST)] into the bloodstream and extracellular space ${ }^{1,2)}$. When direct muscle injury or cellular energy depletion occurs, the result is excessive intracellular influx of ionized sodium and calcium. The prolonged presence 
of a high concentration of ionized calcium leads to sustained myofibrillar contraction, ionized calcium-dependent proteases promoting the lysis of the cellular membrane, finally leading to necrosis of muscle fibers and release of muscle contents into the extracellular space and blood stream ${ }^{3}$.

Unlike in adults, an estimated 26,000 rhabdomyolysis cases are reported annually in the US, date on rates are limited in pediatric population. Common causes of rhabdomyolysis in children include infection, physical exertion, drugs, toxins, metabolic disorder. Typically, but not universally, symptoms include myalgia and dark (tea-colored) urine. Alternatively, patients may have nonspecific systemic complaints such as fever, malaise, nausea, and vomiting.

Rhabdomyolysis ranges from an asymptomatic illness to a life-threatening condition associated with extreme elevation in $\mathrm{CK}$, electrolyte imbalance, acute kidney injury (AKI), cardiac arrhythmias, compartment syndrome, and disseminated intravascular coagulation (DIC). The diagnosis can be confirmed using serum myoglobin, but increase in myoglobin concentration tends to normalize within 6 to 8 hours following muscle injury. Using serum myoglobin level is not as sensitive as using CK because of the former's rapid renal clearance, and false-negative results may occur based on the timing of measurements. Although a cutoff threshold has not been established for the diagnosis, CK activity of at least 5 times the upper limit of the reference range is commonly used ${ }^{4-7)}$.

The goals of management in a patient with rhabdomyolysis are 2-fold: treatment of the underlying cause of the illness and avoidance of kidney function deterioration. Fluid therapy increases renal perfusion, inhibits cast formation, and prevents further ischemic damage to the kidney. $\mathrm{Al}$ though no specific management guidelines exist for pediatric cases, initial fluid resuscitation may include correcting hypovolemia using 10 to $20 \mathrm{~mL} / \mathrm{kg}$ fluid boluses as needed ${ }^{8)}$.

Case reports that describe risk factors related to rhabdomyolysis, underlying diseases, mortality, and AKI in Korean pediatric patients are scarce. Thus, in this study, we investigated the cause and clinical manifestations of rhabdomyolysis, and analyzed its prevalence using available clinical data.

\section{Materials and methods}

We retrospectively reviewed the medical records of patients with rhabdomyolysis who were treated at the pediatric clinic of Pusan National University Children's Hospital from January 2011 to July 2016. The diagnostic criteria for rhabdomyolysis were serum myoglobin of $\geq 80 \mathrm{ng} / \mathrm{mL}^{9,10)}$ and the absence of acute myocardial injury, cardiac arrest, and brain damage. CK, serum myoglobin, aspartate transaminase, lactate dehydrogenase, blood urea nitrogen, creatinine, initial and peak CK, and serum myoglobin levels were measured at the time of admission or at the time of diagnosis. Urine myoglobin, occult blood, and red blood cells were examined. Based on medical records, sex, age at diagnosis, underlying disease, gross hematuria, myalgia, treatment methods, and complications were investigated. In the case of rhabdomyolysis occurred by two or more infections, all infections were recorded. The definition of AKI was based on specific age-standardized reference ranges in children. Descriptive statistics were used to describe the basic features of the data in this study. T test and chi square test were used to compare parameters in between AKI patients and non-AKI patients.

\section{Results}

Forty-five patients were enrolled, the mean age was $116 \pm$ 68 months (median, 120 months; range, 8-264 months); two-thirds of the patients experienced myalgia and/or gross hematuria (Table 1). Initial CK was 20,480 $557,106 \mathrm{U} / \mathrm{L}$ and the peak CK level was $24,072 \pm 65,312 \mathrm{U} / \mathrm{L}$ during the hospitalization period. The initial myoglobin was $963 \pm 1,559 \mathrm{ng} /$

\begin{tabular}{|c|c|}
\hline & No. $=45(\%)$ \\
\hline Age (months) & $116 \pm 68$ \\
\hline \multicolumn{2}{|l|}{ Gender } \\
\hline Male & $35(77.7)$ \\
\hline Female & $10(22.2)$ \\
\hline \multicolumn{2}{|l|}{ Initial symptoms } \\
\hline Myalgia+Gross hematuria & $7(15.5)$ \\
\hline Myalgia only & $19(42.2)$ \\
\hline Gross hematuria only & $5(11.1)$ \\
\hline No symptom & $14(31.1)$ \\
\hline
\end{tabular}


$\mathrm{mL}$ and the peak myoglobin level was $985 \pm 1,558 \mathrm{ng} / \mathrm{mL}$. Among 16 patients with positive occult blood test, 10 patients had true hematuria (both urine occult blood and red blood cells were positive) except one patient who did not undergo urine screening (Table 2).

The most common cause of rhabdomyolysis was infection; Influenza virus was more commonly implicated than other viral infections, such as parainfluenza. Physical exertion was the second most common cause (20\%); and included punishment, excessive exercise, spinning, weight training, badminton, and running. Three patients had rhabdomyolysis due to metabolic disorders, and were diagnosed with long-chain 3-hydroxyacyl-coA dehydrogenase deficiency, neuroleptic malignant syndrome, and duchenne muscular dystrophy. Two patients were diagnosed with rhabdomyolysis due to drug addiction, and the causative drug was aripiprazole and diphenhydramine (Table 3). Seasonal distribution of rhabdomyolysis occurrence showed a high distribution in winter (December-February) and summer (June-August).

No complications in 35 patients (77.7\%), but complications occurred in 10 patients (22.2\%), including acute kidney injury in 6 patients (13.3\%) and electrolyte abnormalities in patients without acute kidney injury (2.2\%), two of whom had acute kidney injury associated with DIC (Table 4).

In most cases, rhabdomyolysis was treated by administering more fluid than the maintenance dose (generally, double maintenance dose). Two patients were treated by additional alkalization. In two patients, acute kidney injury progressively worsened and hemodialysis was performed.

\begin{tabular}{lr} 
Table 2. Laboratory Finding \\
\begin{tabular}{lr}
\hline Laboratory test \\
\hline CK (U/L)
\end{tabular} \\
Initial & $20,480 \pm 57,106$ \\
Peak & $24,072 \pm 65,312$ \\
Myoglobin (ng/mL) & \\
Initial & $963 \pm 1,559$ \\
Peak & $985 \pm 1,558$ \\
AST (IU/L) & $736 \pm 2,979$ \\
Urine myoglobin (+) & $10 / 31(32.2)$ \\
Urine OB (+) & $16 / 44(36.3)$ \\
Urine RBC (-) & $6(13.6)$ \\
Urine RBC (+) & $10(22.7)$ \\
\hline
\end{tabular}

Abbreviations: CK, creatine kinase; AST, aspartate aminotransferase; $O B$, occult blood; RBC, red blood cell.
(Table 5). These patients had lived with nephrotic syndrome for a long time and at the time of rhabdomyolysis onset, were experiencing relapse.

Table 6 shows a comparison of the clinical characteristics and laboratory findings of AKI and non-AKI patients. Gross hematuria, positive occult blood test, and positive urine protein were more common in the AKI group than in the non-AKI group. Initial and peak CK and serum myoglobin levels were non-significantly higher in the AKI group than in the non-AKI

Table 3. Causes of Rhabdomyolysis

\begin{tabular}{lc}
\hline Cause & No. of cases (\%) \\
\hline Infection & $26(57.7)$ \\
Influenza & 11 \\
Parainfluenza & 2 \\
Coronavirus & 1 \\
Adenovirus & 1 \\
Rhinovirus & 1 \\
Enterovirus & 1 \\
Mycoplasma & 2 \\
\hline Unknown & 9 \\
Physical exertion & $9(20)$ \\
Prolonged seizure & $1(2.2)$ \\
Metabolic abnormalities & $3(6.6)$ \\
Medication & $2(4.4)$ \\
Multiorgan failure (near drowing) & $4(8.8)$ \\
\hline
\end{tabular}

Table 4. Complications

\begin{tabular}{lc}
\hline Complications & No. (\%) \\
\hline Acute kidney injury & $6(13.3)$ \\
Compartment syndrome & 0 \\
DIC & $5(11.1)$ \\
Electrolyte abnormalities & $1(2.2)$ \\
None & $35(77.7)$ \\
\hline Abbreviations: DIC, disseminated intravascular coagulation.
\end{tabular}

Table 5. Treatment and Outcome

\begin{tabular}{lc}
\hline & No. (\%) \\
\hline Hydration only & $37(82.2)$ \\
Alkalization only & 0 \\
Mannitol only & 0 \\
Hydration+Alkalization & $2(4.4)$ \\
Hydration+mannitol & 0 \\
Mannitol+alkalization & 0 \\
Hydration+Mannitol+Alkalization & 0 \\
Dialysis & $2(4.4)$ \\
No specific therapy & $4(8.8)$
\end{tabular}




\section{Discussion}

This study was designed to investigate the clinical features of pediatric rhabdomyolysis in a single tertiary center in Korea. In children, rhabdomyolysis can occur at all ages, but in this study, the most frequent distribution was observed between 10 - and 15-year-olds. A study by Kim et al. described a high incidence of rhabdomyolysis in children of ages 1 to 5 years, which is different from the age range in our study. Incidence, morbidity, and mortality were higher in male (adults and children) patients than in female patients ${ }^{11)}$.

Although, no diagnostic criteria for rhabdomyolysis exist, most clinicians indicate CK levels 5 times higher than the normal range. However, the cutoff threshold is not clearly established. Serum and urine myoglobin can also be considered in the diagnosis. The half-life of CK is 1.5 days, while that of serum myoglobin is 2 to 4 hours, normalization of increased myoglobin concentration occurs after 6 to 8 hours. Serum myoglobin is thought to be golden standard for diagnosis, but is not as sensitive as $\mathrm{CK}$ for diagnosis because of its short half-life, resulting in false-negative tests ${ }^{3,6}$. In this study, we defined rhabdomyolysis as an elevated serum myoglobin, because we intended to confirm patient for a more clear study of pediatric rhabdomyolysis.

The cause of rhabdomyolysis may also vary widely. Although trauma and drugs are the most common causes of rhabdomyolysis in adults, in children, common causes of sporadic rhabdomyolysis are viral myositis, trauma, exces- sive exercise, drug overdose, and neuroleptic malignant syndrome $^{12}$. A study by Park JS et al., which involved Korean adults, in comparison with previous studies that involved adults, presented the most common cause of rhabdomyolysis, as substance abuse (35\%) followed by direct muscle injury (31\%), such as over-activity, immobilization, and trauma. The incidence of infection was very low at $3 \%$, which is inconsistent with our study where infection and trauma were $56.2 \%$ and $18 \%$ respectively ${ }^{13)}$. According to a study by Rodríguez E et al., in $27.8 \%$ of patients, drugs (heroin, cocaine, narcotics, alcohols), infectious diseases, including pneumonia (19.8\%) and trauma (7.1\%) were the causes of rhabdomyolysis. In adults, the proportion of drug-induced rhabdomyolysis was high which makes it different from the situation in children ${ }^{14)}$.

Unlike in adults, infection is the major cause of rhabdomyolysis in children; especially, influenza virus. In 2009 H1N1 influenza virus pandemic, several rhabdomyolysis cases of varying severity were published. Kim JH et al. had similar results. Of the causes of rhabdomyolysis, the percentages of pneumonia and acute respiratory distress syndrome, seizures, and excessive exercise, were 15\%, 11.6\%, and $3.3 \%$, respectively, and that of infection was high. The rate of rhabdomyolysis due to physical exertion was significantly lower than that of the present study ${ }^{15)}$. Most reports of influenza-associated rhabdomyolysis involve influenza type A virus in children, and there are some reports on benign acute childhood myositis associated with type B but reports of influenza B-associated rhabdomyolysis are

Table 6. Comparison Clinical Characteristics and Lab Finding of AKI Group with Non-AKI Group

\begin{tabular}{lccc}
\hline Variable & AKI $(\mathrm{n}=6)$ & Non-AKI $(\mathrm{n}=39)$ & $P$-value \\
\hline Age (month) & $148 \pm 86$ & $111 \pm 65$ & 0.230 \\
Myalgia & 2 & 24 & 0.201 \\
Gross hematuria & 4 & 8 & 0.017 \\
Urine OB (+) & 6 & 10 & $<0.001$ \\
Urine RBC $(+)^{*}$ & 3 & 1 & 0.088 \\
Urine protein $(+)^{\dagger}$ & 5 & 14 & 0.038 \\
CK (initial, IU/L) & $41,457 \pm 86,581$ & $17,253 \pm 52,053$ & 0.340 \\
CK (peak, IU/L) & $44,722 \pm 85,964$ & $20,896 \pm 62,363$ & 0.412 \\
Serum myoglobin (initial, ng/mL) & $2,553 \pm 2,919$ & $719 \pm 1,111$ & 0.186 \\
Serum myoglobin (peak, ng/mL) & $2,624 \pm 2,890$ & $732 \pm 1,106$ & 0.171 \\
AST (IU/L) & $3,819 \pm 7,977$ & $262 \pm 420$ & 0.325 \\
LDH (IU/L) & $9,424 \pm 17,521$ & $1,446 \pm 1,838$ & 0.316 \\
\hline
\end{tabular}

*Urine RBC positive : $>5 / \mathrm{HPF}$.

${ }^{\dagger}$ Urine protein positive : $>10 \mathrm{mg} / \mathrm{dL}$. 
$\operatorname{rare}^{16)}$. In 2010, Wu CT et al. retrospectively reviewed the records of children who visited a university children's hospital in North Taiwan for influenza B infection between 2000 and 2007, and found that 24 children presented with rhabdomyolysis. These children recovered without complications during the periods of study follow up ${ }^{17)}$. In this study, 11 patients were infected with influenza virus and all of them were treated with appropriated fluid therapy without complications, including AKI. This is similar to previous reports. In addition to influenza viruses, rotavirus ${ }^{18)}$, varicella ${ }^{19)}$, Epstein-barr virus ${ }^{20)}$, cytomagelovirus ${ }^{21)}$, herpes simplex ${ }^{22)}$, and human immunodeficiency virus ${ }^{23)}$ have also been reported to cause rhabdomyolysis in children. Mycoplasma pneumoniae has been rarely reported in an adult population, and even more uncommonly in children. Rhabdomyolysis and its proposed mechanisms in patients with mycoplasma pnemoniae infection are poorly understood but are associated with potentially serious complications ${ }^{24)}$. In our study, two patients with rhabdomyolysis associated with mycoplasma pneumonia were presented. Similar to the previous case study, appropriate fluid therapy and clarithromycin, without other therapies, led to the patient's recovery without complications.

Although substance abuse is the major cause of rhabdomyolysis in adults, two cases of drug-induced rhabdomyolysis were also found in this study. A 14-year-old girl diagnosed with schizophrenia and another patient who was taking aripiprazole. The possible mechanism of rhabdomyolysis related to aripiprazole was the effect of antagonist activity at serotoin 2A (5-HT2A) receptors in skeletal muscles. This drug has been reported in 76 cases of overdose and in 10 pediatric ( 12 years or less) cases, and is associated with clinically important symptoms, which may include metabolic acidosis, elevation of $\mathrm{CK}$, alanine transaminase, convulsions, and hypokalemia ${ }^{25)}$. Rhabdomyolysis associated with antipsychotic use is a rare occurrence. The rarity and unspecific early symptoms of rhabdomyolysis might contribute to the difficulty in recognizing its manifestation due to drug use ${ }^{26,27)}$. The other patient was an 18-year-old girl with no reliable medical history who was diagnosed with rhabdomyolysis after she ingested a large dose of diphenhydramine in a suicide attempt. Although there is no clear mechanism regarding the effect of diphenhydramine on rhabdomyolysis onset, the patient was treated through fluid therapy without secondary complications $^{28)}$.

AKI is a major life-threatening complication of rhabdomyolysis that requires immediate adequate management, most patients improved by massive hydration without complication. However, several pathophysiological mechanisms have been investigated in AKI, including hypovolemia, resulting in decreased renal perfusion, renal tubular obstruction by myoglobin cast formation, and direct toxic effects of myoglobin. Few studies have examined predictors for the development of AKI in patients with rhabdomyolysis. The levels of CK, myoglobin, and peak levels of CK have been used as an appropriate index for diagnosis and severity, predictive factor of AKI. Previous studies reported that the rate of AKI-associated rhabdomyolysis ranges from $42-50 \%{ }^{29,30}$. However, recent larger pediatric studies showed that the rate of AKI associated with pediatric rhabdomyolysis ranged from 8.7 to $-35.9 \%{ }^{1,4,31)}$. Our study shows that the rate of AKI was 13.3\%, which was much lower than those of previous reports. The differences in the incidence of rhabdomyolysis in these studies might be attributed to the characteristics of the underlying disease and time of taking treatment for the illness.

Limitations of this study include the small patient population in a single center and retrospective nature of the study. Another limitation is that as we defined AKI based on specific age-standardized reference ranges in children, it may be possible that the prevalence of AKI lower than the actual rate.

In summary, rhabdomyolysis in children was caused by infection, physical exertion, seizure and metabolic problems. Although complications occurred in about $20 \%$ of our study population, most patients with complications recovered completely through sufficient fluid therapy. However, two patients with nephrotic syndrome relapse had to be treated using dialysis for AKI in the present study. In patients with underlying kidney disease, careful treatment is needed, and renal replacement therapy should be considered.

\section{Ethics statement}

This study was approved by Pusan National University 
Yangsan hospital's Institutional Review Board (IRB No. 05-2018-156).

\section{Conflicts of interest}

\section{No potential conflict of interest relevant to this article was reported.}

\section{References}

1. Kwiatkowski DM, Sutherland SM. Acute kidney injury in pediatric patients. Best Pract Res Clin Anaesthesiol 2017;31:427-39.

2. You JH, Lee JN, Park YS, Lee JH. Virus-associated Rhabdomyolysis in Children. Child kidney Dis 2017;21:89-93.

3. Essam FE, Robert FR. Rhabdomyolysis: a review, with emphasis on the pediatric population. Pediatr. Nephrol 2010;25:7-18.

4. Chen CY, Lin YR, Zhao LL et al. Clinical spectrum of rhabdomyolysis presented to pediatric emergency department. BMC Pediatr 2013;13:134.

5. Luck RP, Verbin S. Rhabdomyolysis: A review of clinical presentation, etiology, diagnosis, and management. Pediatr. Emerg. Care 2008;24:262-8.

6. Torres PA, Helmstetter JA, Kaye AM, Kaye AD. Rhabdomyolysis: Pathogenesis, Diagnosis, and Treatment. Ochsner J 2015;1:58-69.

7. Zutt R, van der Kooi AJ, Linthorst GE, Wanders RJ, de Visser M. Rhabdomyolysis: Review of the literature. Neuromuscul Disord 2014;24:651-9.

8. Al-Ismaili Z, Piccioni M, Zappitelli M. Rhabdomyolysis: Pathogenesis of renal injury and management. Pediatr Nephrol 2011;26: 1781-8.

9. Kasik JW, Leuschen MP, Bolam DL, Nelson RM. Rhabomyolysis and myoglobinemia in neonates. Pediatrics 1985;76:255-8.

10. Chapelle JP, Lemache K. Fast determination of myoglobin in serum using a new radial partition immunoassay. Clin Biochem 1994;27:423-8

11. Singh U, Scheld WM. Infectious etiologies of rhabdomyolysis: Three case reports and review. Clin Infect Dis 1996;22:642-9.

12. Melli G, Chaudhry V, Cornblath DR. Rhabdomyolysis: An evaluation of 475 hospitalized patients. Medicine (Baltimore) 2005;84: 377-85.

13. Park JS, Seo MS, Gil HW, Yang JO, Lee EY, Hong SY. Incidence, etiology, and outcomes of rhabdomyolysis in a single tertiary referral center. J Korean Med Sci 2013;28:1194-9.

14. Rodríguez E, Soler MJ, Rap O, Barrios C, Orfila MA, Pascual J. Risk factors for acute kidney injury in severe rhabdomyolysis. PLoS
One 2013;8:8-13.

15. Kim JH, Goo MJ, Yeom JS, Park ES, Seo JH, Lim JY. Clinical characteristics of acute renal failure of rhabdomyolysis in children 2007; 50:277-83.

16. Ghoshh JB. Influenza-B associated rhabdomyolysis and acute renal failure. Indian Pediatr 2013;50:595-6.

17. Wu CT, Hsia SH, Huang JL. Influenza B-associated rhabdomyolysis in Taiwanese children. Acta Paediatr 2010;99:1701-4.

18. Minami K, Tamura A, Komori Y, Yanagawa T, Izumi G, Kioka N, Yoshikawa N. Acute encephalopathy and rhabdomyolysis following rotavirus gastroenteritis. J Paediatr Child Health 2007;43: 90-1.

19. Pratt RD, Bradley JS, Loubert C, LaRocco A Jr, McNeal RM, Newbury RO, Sawyer MH. Rhabdomyolysis Associated with Acute Varicella Infection. Clin Infect Dis 1995;20:450-3.

20. Friedman BI, Libby R. Epstein-Barr Virus Infection Associated with Rhabdomyolysis and Acute Renal Failure. Clin Pediatr (Phila) 1986;25:228-9.

21. Gindre H, Féasson L, Auboyer C, Cathébras P. Severe rhabdomyolysis associated with a primary cytomegalovirus infection in an immunocompetent patient. BMJ Case Rep 2013.

22. Mackay MT, Kornberg AJ, Shield LK, Dennett X. Benign acute childhood myositis: laboratory and clinical features. Neurology 1999;53:2127-31.

23. Chariot P, Ruet E, Authier FJ, Lévy Y, Gherardi R. Acute rhabdomyolysis in patients infected by human immunodeficiency virus. Neurology 1994;44:1692-6.

24. Jensen JS, Heilmann C, Valerius NH. Mycoplasma pneumoniae infection in a child with AIDS. Clin Infect Dis 1994;19:207.

25. Akhtar NM, Khan AB. Prescriptions into practice : Aripiprazole (Abilify): A Novel Atypical Antipsychotic Medication. Child and Adolescent Psychopharmacology News 2008;13:1-4

26. Chang KY, Wu YF. Aripiprazole-associated rhabdomyolysis in a patient with schizophrenia. J Neuropsychiatry Clin Neurosci 2011;22:E5123.

27. Star K, lessa N, Almandil NB, Wilton L, Curran S, Edwards IR, Wong IC. Rhabdomyolysis Reported for Children and Adolescents Treated with Antipsychotic Medicines: A Case Series Analysis. J Child Adolesc Psychopharmacol 2012;22:440-51.

28. Emadian SM, Caravati EM, Herr RD. Rhabdomyolysis: A rare adverse effect of diphenhydramine overdose Am J Emerg Med 1996;14:574-6.

29. Bosch X, Poch E, Grau JM. Rhabdomyolysis and Acute Kidney Injury. N Engl J Med 2009;361:62-72.

30. Watemberg N, Leshner RL, Armstrong BA, Lerman-Sagie T. Acute pediatric rhabdomyolysis. J Child Neurol 2000;15:222-7.

31. Wu CT, Huang JL, Lin JJ, Hsia SH. Factors associated with nontraumatic rhabdomyolysis and acute renal failure of children in Taiwan population. Pediatr Emerg Care 2009;25:657-60. 